\title{
MS08-P06 | Crystal Structure Of UHRF1:Lig1 Complex ReVealed Structural \\ Change Of Uhrf1 And The Key Residues For High Affinity Binding
}

Kori, Satomi (Graduate School of Medical Life Science, Yokohama City University, Yokohama, JPN); Ferry, Laure (Univ. Paris Diderot, Sorbonne Paris Cité, Epigenetics and Cell Fate, Paris, FRA); Matano, Shohei (Graduate School of Medical Life Science, Yokohama City University, Yokohama, JPN); Kodera, Noriyuki (Bio-AFM Frontier Research Center, Kanazawa University, Kanazawa, JPN); Oda, Takashi (Graduate School of Medical Life Science, Yokohama City University, Yokohama, JPN); Defossez, Pierre-Antoine (Univ. Paris Diderot, Sorbonne Paris Cité, Epigenetics and Cell Fate, Paris, FRA); Arita, Kyohei (Graduate School of Medical Life Science, Yokohama City University, Yokohama, JPN)

DNA methylation is an important epigenetic modification, which involved in development, X-chromosome inactivation, genome imprinting, and carcinogenesis. DNA methylation patterns are re-established after DNA replication and UHRF1 plays an essential role in this process. Tandem Tudor Domain (TTD) of UHRF1 is critical for its function. It binds various partners by intra or inter-molecule interaction; histone H3K9me2/3, linker 2 or spacer. Recently, we reported that a methylated histone-like region of DNA Ligase1 (LIG1K126me2/3) binds to the UHRF1 TTD. We also revealed that the affinity of LIG1K126me3 binding to TTD is higher than that of H3K9me3. However, the underlying mechanism by which the binding of LIG1K126me3 to TTD with high affinity is unclear. Here, we show the crystal structure of the UHRF1 TTD in complex with LIG1K126me3 peptide. Mutational studies revealed the key residues for high affinity binding of LIG1K126me3 and that the interaction probably regulated by phosphorylation of LIG1. Furthermore, SAXS and high-speed AFM analysis indicated that the binding of LIG1K126me3 changes the overall structure of UHRF1 to flexible. These results suggest that LIG1K126me 3 is an allosteric regulator that modulates the conformation of UHRF1 in a different manner from H3K9me3. We will also discuss development of UHRF1 targeting inhibitor that compete with the binding of LIG1. 